\title{
Self-narrative reconstruction after dilemma-focused therapy for depression: A comparison of good and poor outcome cases
}

\author{
ADRIÁN MONTESANO ${ }^{1,2}$, MIGUEL M. GONÇALVES ${ }^{3}$, \& GUILLEM FEIXAS ${ }^{1,2}$ \\ ${ }^{1}$ Department of Personality, Assessment and Psychological Treatments, University of Barcelona, Barcelona, Spain; ${ }^{2}$ Institute for \\ Brain, Cognition and Behavior, University of Barcelona, Barcelona, Spain $\mathcal{E}^{3}$ School of Psychology, University of Minho, \\ Braga, Portugal
}

\begin{abstract}
Objective: The aim of this study is to improve the understanding of self-changes after an intervention for depression focused on implicative dilemmas, a type of cognitive conflict related to identity. As recent research has highlighted the relevance of identity-related dilemmas in clients with depression, we sought to assess the way in which clients resolve such inner conflicts after a tailored dilemma-focused intervention and how this is reflected in the clients' self-narratives. Method: We used three instruments to observe differences between good $(n=5)$ and poor $(n=5)$ outcome cases: (i) the Repertory Grid Technique to track the resolution of dilemmas, (ii) the Change Interview to compile clients' accounts of changes at posttreatment, and (iii) the Innovative Moments Coding System to examine the emergence of clients' novelties at the Change Interview. Results: Groups did not differ in terms of the number and relevance of client-identified significantly helpful events. However, between-group differences were found for the resolution of dilemmas and for the proportion of high-level innovative moment (IM) types. Furthermore, a greater self-narrative reconstruction was associated with higher levels of symptom improvement. Conclusions: Good outcome cases seem to be associated with the resolution of conflicts and high-level IMs.
\end{abstract}

Keywords: depression; innovative moments; implicative dilemmas; resistance and ambivalence; self and identity

During the last few decades, several meta-analyses have demonstrated the efficacy of psychotherapy for depression (Barth et al., 2013; Cuijpers, Andersson, Donker, \& Van Straten, 2011). The mediation processes involved in the treatment of depression have also received extensive support from a growing body of research on change mechanisms, especially from a variety of cognitive psychotherapies for depression (Dozois \& Dobson, 2001; Garrat, Ingram, Sawalani, \& Rand, 2007; Hollon, 2006). Nevertheless, the precise mechanisms that underlie the efficacy of cognitive and other forms of therapy are not presently known (see Basto \& Salgado, 2014; for a review).

Although most cognitive models for depression sustain that change in "deep" self-schemas is the key factor for therapeutic improvement, most of the research about the mechanism of change focuses on "surface" cognitions (e.g., automatic thoughts, attribution bias). This is probably due to the difficulty of tapping deeper self-schemas. With this challenge in mind, a research line has focused on the study of the role of implicative dilemmas (IDs), a conflictual self-schema in which symptoms are associated with core personal values (see below for a detailed description), which aims to clarify the cognitive processes that are involved in the maintenance of depressive symptoms. In particular, a series of studies found that IDs were significantly more prevalent in depressive participants than in controls and that such cognitive conflicts were associated with the severity of patients' clinical complaints (Feixas, Montesano,

Correspondence concerning this article should be addressed to Adrián Montesano, Department of Personality, Assessment and Psychological Treatments, University of Barcelona, Barcelona, Spain. Email: adrianmontesano@gmail.com 
Compañ, et al., 2014; Feixas, Montesano, ErazoCaicedo, Compañg, \& Pucurull, 2014; Montesano et al., 2014). Consequently, a dilemma-focused intervention was devised to target IDs (Feixas \& Compañ, 2015) and is currently being tested for the treatment of depression in combination with standard CBT (Feixas et al., 2013). Preliminary analyses (Feixas et al., 2015) showed promising results for dilemma-focused therapy, which proved to be at least as effective as standard CBT in improving depressive symptoms and more suitable for helping clients resolving their IDs.

Based on this ongoing study, for the present article, we sought to improve our understanding of change after dilemma-focused therapy for depression. We compared the therapeutic change of good and poor outcome cases, paying special attention to clients' self-narratives to gain a better understanding of the processes that are involved in symptom improvement and in conflict resolution. In doing so, we analyzed clients' self-narrative reconstructions by exploring the resolution of conflicts and client profiles of innovative moments (IMs) after therapy.

As we explain below, IMs are markers of meaning transformation in self-narratives that are associated with change. Specifically, the concept of IMs refers to the emergence of novelties that challenge the dominant problematic self-narrative (Gonçalves, Matos, \& Santos, 2009) or maladaptive pattern that brought the client to therapy (including feelings, thoughts, behaviors, and relationships). In this study, we did not track the emergence of IMs throughout the therapeutic process, as most studies with IMs used to do. Instead, we inspected the client profiles of IMs in a posttreatment Change Interview (Elliott, Slatick, \& Urman, 2001) to capture the level of self-narrative reconstruction achieved after treatment. Additionally, we analyzed the number and relevance of changes perceived by the clients at such interview.

\section{IDs and Depression}

In brief, Kelly's (1955/1991) theory is based on the analysis of personal constructs, which are bipolar dimensions of personal meaning (e.g., altruist vs. selfish; strong vs. weak). He suggested that each individual develops a unique but limited set of personal constructs that are hierarchically organized within an evolving, interconnected personal system. The notion of ID (see Feixas, Saúl, \& Ávila, 2009) draws from personal construct theory and defines a particular type of conflict between two opposing parts of the self (two personal constructs). Specifically, an ID is a conflict in which a desired change (e.g., taking better care of oneself) simultaneously implies an unwished change on a core aspect of a client's identity (e.g., to stop being altruistic and become more selfish). This implication (e.g., associating taking care of oneself with being selfish) is based on an implicit association between the two personal constructs. The net aftermath of the clash between these two opposing forces often results in a vacillation that could paralyze clients from achieving their desired changes. Operationally, there are three main elements involved in an ID (see Figure 1):

(1) Congruent constructs refer to the need for continuity and personal coherence. They are constructs in which a person perceives congruency between the present self and the ideal self. These are mostly core constructs that are connected to personal values, beliefs, and attitudes that define the central sense of an individual's identity (Montesano et al., 2014; Montesano, Feixas, \& Varlotta, 2009); therefore, a person would resist changing to avoid self-invalidation. In the example, both the actual self and the ideal self of the participant are regarded as "altruistic" as opposed to "selfish."

(2) Discrepant constructs refer to the need for change. They are self-representations in which a person perceives a meaningful discrepancy between the present self and the ideal self. This type of construct represents perceived negative attributes, which typically indicate areas of malaise and symptoms in which a change from one pole to the opposite pole of the construct is desired. In Figure 1, the present self is in the pole, "not take care of myself", and the ideal self is in the pole, "take care of myself."

(3) An association between these two constructs within a client's personal construct system in such a way that the desired change implies an unwished change, as in the example in which "taking care of one self" implies "becoming selfish."

Note that it is neither the self-ideal discrepancy nor the self-ideal congruency per se that poses a dilemmatic conflict to an individual. Rather, it is the implicit association between the two constructs that causes conflict. The implicit nature of IDs determines that the person is often not aware of the existence of such cognitive conflict.

An ID, therefore, is identified whenever there is a significant correlation between the desired pole of the discrepant construct (taking care of myself) and the undesired pole of the congruent construct 


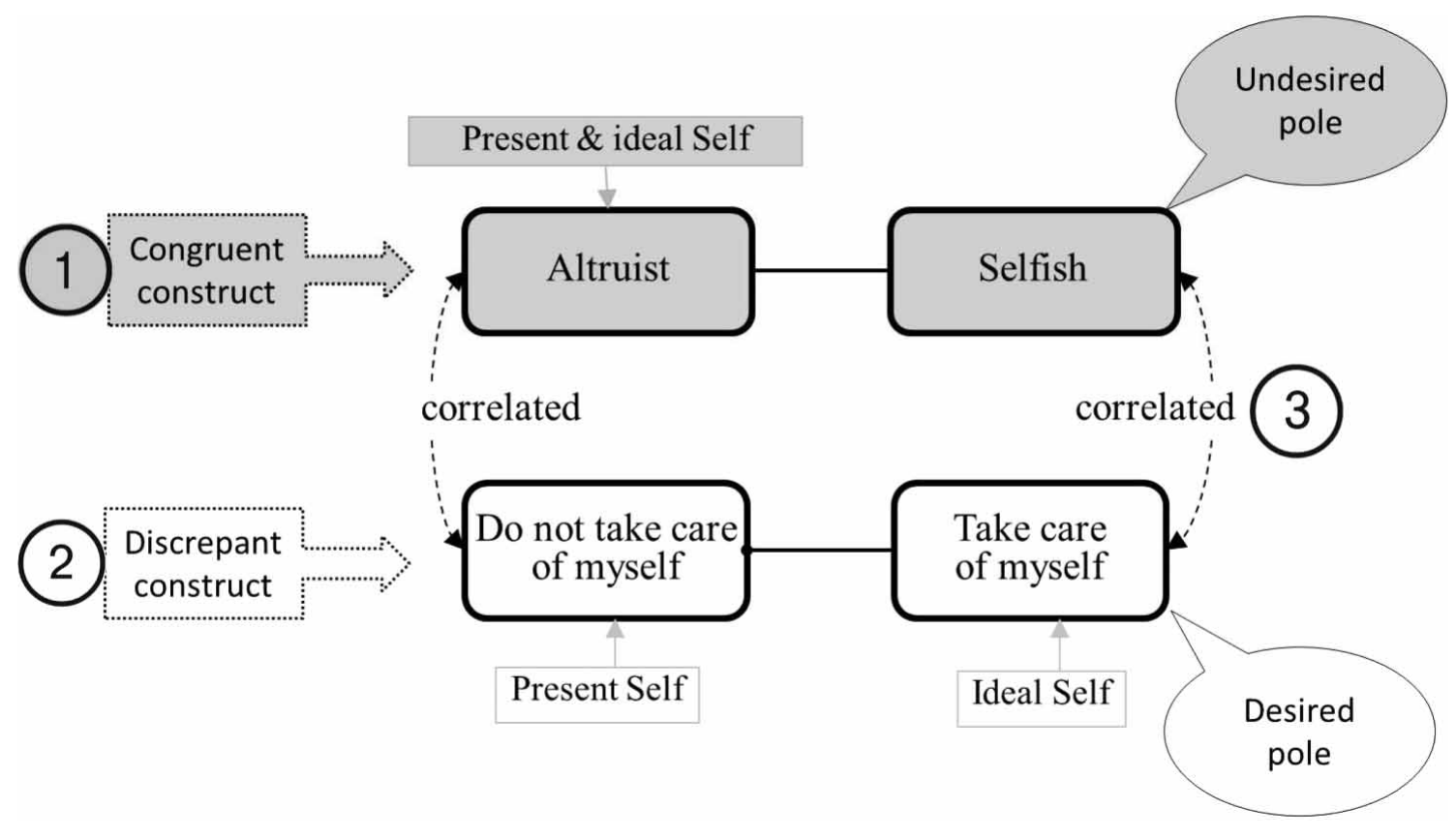

Figure 1. Components of an ID from a participant of the study.

(becoming selfish). In the example of Figure 1, the client presents the dilemma of choosing between being the type of person who "is altruistic but does not take care of herself" or who "takes care of herself but becomes selfish," given the implicit association between the poles of the constructs. Therefore, in this case, the need for change (taking better care of herself) might be hindered by the need to maintain self-ideal congruency and to avoid the invalidation of core aspects of the self (stopping being altruistic and becoming selfish).

Note that the content of these discrepant and congruent constructs is not defined in advance by the researcher or therapist. Rather, constructs are personal and therefore derived from a client's way of construing. Another client might present an ID with different specific content. For instance, another female participant in the study presented an ID that implied the dilemmatic proposition of unknowingly choosing between "being a good person but depressed" or "being an equilibrated but bad person," In this example, the association between the symptoms' need and a core personal value is clear. In particular, the client's ID involved the association of the discrepant construct "depressed (present self) vs. equilibrated (ideal self)" with the congruent construct "good person (present and ideal self) vs. bad person." These two bipolar constructs were correlated in that being depressed was associated in the person's mind with goodness, whereas being equilibrated was associated with badness. The present self was constructed as being a good person but depressed. However, whereas goodness was congruent with the self, being depressed was discrepant, and therefore there was a wish for change toward the opposite, namely, being equilibrated. Nevertheless, becoming more equilibrated also implied stopping being a good person (thus becoming a worse person), which supposed a strong invalidation of the client's sense of identity, which therefore led to an identity-related cognitive conflict. The worst consequence of such conflict is that it might hinder the participant's attempts to improve her depressive symptoms, given that she would unwittingly prefer being a good person but depressed, rather than being equilibrated and therefore a bad person, to preserve personal coherence.

In sum, we assume that IDs (i) are conflicts among opposing parts of the self (discrepant and congruent constructs) that could lead to ambivalence and resistance to change (see also Engle \& Arkowitz, 2008; Gonçalves, Ribeiro, Conde, et al., 2011), (ii) are often out of a client's awareness, and (iii) have personal meanings that serve as a protective function against invalidation of core aspects of identity.

A series of studies found consistent evidence for the relevance of IDs in major depression and dysthymia, indicating significant differences between depressed and non-depressed individuals with regard to the presence and level of IDs (Feixas, Montesano, Compañ, et al., 2014; Feixas, Montesano, Erazo-Caicedo, et al., 2014; Montesano et al., 2014). In addition, the level of conflict was associated with the severity of symptoms, poor global 
functioning, and increased risk of suicide (Feixas, Montesano, Compañ, et al., 2014). Furthermore, the level of conflict predicted the same depressive clinical status as predicted by negative self-views, which indicates that IDs play a noticeable role in the identity of depressive individuals (Montesano, Feixas, \& Winter, 2014). These results of the current study are in line with the idea suggested in the bestseller by Dorothy Rowe (1983), which noted that depressive clients often embrace the dilemmatic proposition "I'd rather be good than happy" in their self-narratives (Montesano et al., 2014).

There is already some evidence that indicates that the resolution of IDs is associated with better outcomes in psychotherapy in naturalistic settings (Feixas, Saúl, Winter, \& Watson, 2008; Montesano, Feixas, Muñoz, \& Compañ, 2014). As stated before, there is also promising preliminary evidence for dilemma-focused therapy in the treatment of depression (Feixas et al., 2015), showing a large effect size in symptom reduction $(d=1.36)$. However, to the extent of our knowledge, there are no systematic studies on how dilemmas are resolved.

Tracking internal conflicts is nevertheless a difficult task given their implicit nature. Consistent with dilemma-focused intervention, our hypothesis is that the resolution of IDs implies a certain level of self-narrative reconstruction. As represented in Figure 2, IDs could be resolved mainly by (i) the dissolution of the association between discrepant and congruent constructs and (ii) the reduction of the self-ideal discrepancy. Conversely, the self-ideal congruency is expected to be maintained because a slot change in the congruent construct would threaten the sense of personal identity. Therefore, whenever a depressed client presents with an ID, a significant change is expected to be associated with the reconceptualization of the self in a way that allows for the emergence of a non-dilemmatic self-narrative. To investigate this narrative reconstruction, in the present study we analyzed participants' narrative markers of meaning transformation at a posttreatment interview by tracking IMs.

\section{Innovative Moments}

Recent research on change processes within a narrative framework has focused on understanding the process of self-narrative reconstruction in psychotherapy by analyzing the emergence of IMs (Gonçalves, Ribeiro, Mendes, Matos, \& Santos, 2011; Gonçalves et al., 2009; Ribeiro, Bento, Gonçalves, \& Salgado, 2010). IMs are akin to what is known as exceptions in solution-focused therapy (e.g., de
Shazer, 1985), unique outcomes in narrative therapy (White \& Epston, 1990), or contrary evidence in personal construct therapy (Kelly, 1969). Specifically, IMs refer to instances in which clients challenge their dominant problematic self-narrative by producing an exception to it (Gonçalves et al., 2009; White, 2007; White \& Eptston, 1990). This model of change assumes that the development of an alternative self-narrative follows a process in which the dominant self-narrative is progressively challenged and countered by the emergence of IMs. The authors distinguished five types of IMs: Action, reflection, protest, reconceptualization of the self, and performing change (see Appendix 1).

Results from empirical studies have identified a consistent pattern of change across different therapeutic models and among different clinical conditions. The model holds that whereas action, reflection, and protest can be found in both good and poor outcome cases at the beginning of the therapy, reconceptualization tends to appear in the middle of treatment only in good outcome cases and increases until the end of the treatment, which usually fosters the emergence of performing change IMs. Therefore, the emergence of reconceptualization has been highlighted as a distinctive feature in good outcome cases and is understood to be an essential pathway to clients' development of new, preferred self-narratives. It was hypothesized (Gonçalves \& Ribeiro, 2012) that this type of IM operates as a bridge between the problematic past self-narrative and the emerging, more adjusted self-narrative, which allows a client to assume an agentic position over this change (see the definition of reconceptualization and its dual feature of contrast and process, Appendix 1). Thus, in previous studies (Cunha et al., 2012), action, reflection, and protest IMs were categorized as low-level IMs as they occur in both good and poor outcome cases, and reconceptualization and performing change IMs were categorized as high-level IMs, as they are typical of good outcome cases, particularly after the middle of treatment.

Therefore, high-level IMs can be considered to be markers of self-narrative reconstruction associated with a clinically significant change. Dilemmafocused intervention is intended to promote highlevel IMs because the resolution of IDs implies the re-organization of self-constructions in conflict (congruent and discrepant constructs). In fact, it is expected that some level of self-reconceptualization for resolving IDs is necessary. In this study, we explored clients' self-narrative reconstructions after a tailored dilemma-focused intervention for depression by examining markers of the emergence of a new self-narrative (high-level IMs), contrasting it to the previous dilemmatic self-view (IDs). 


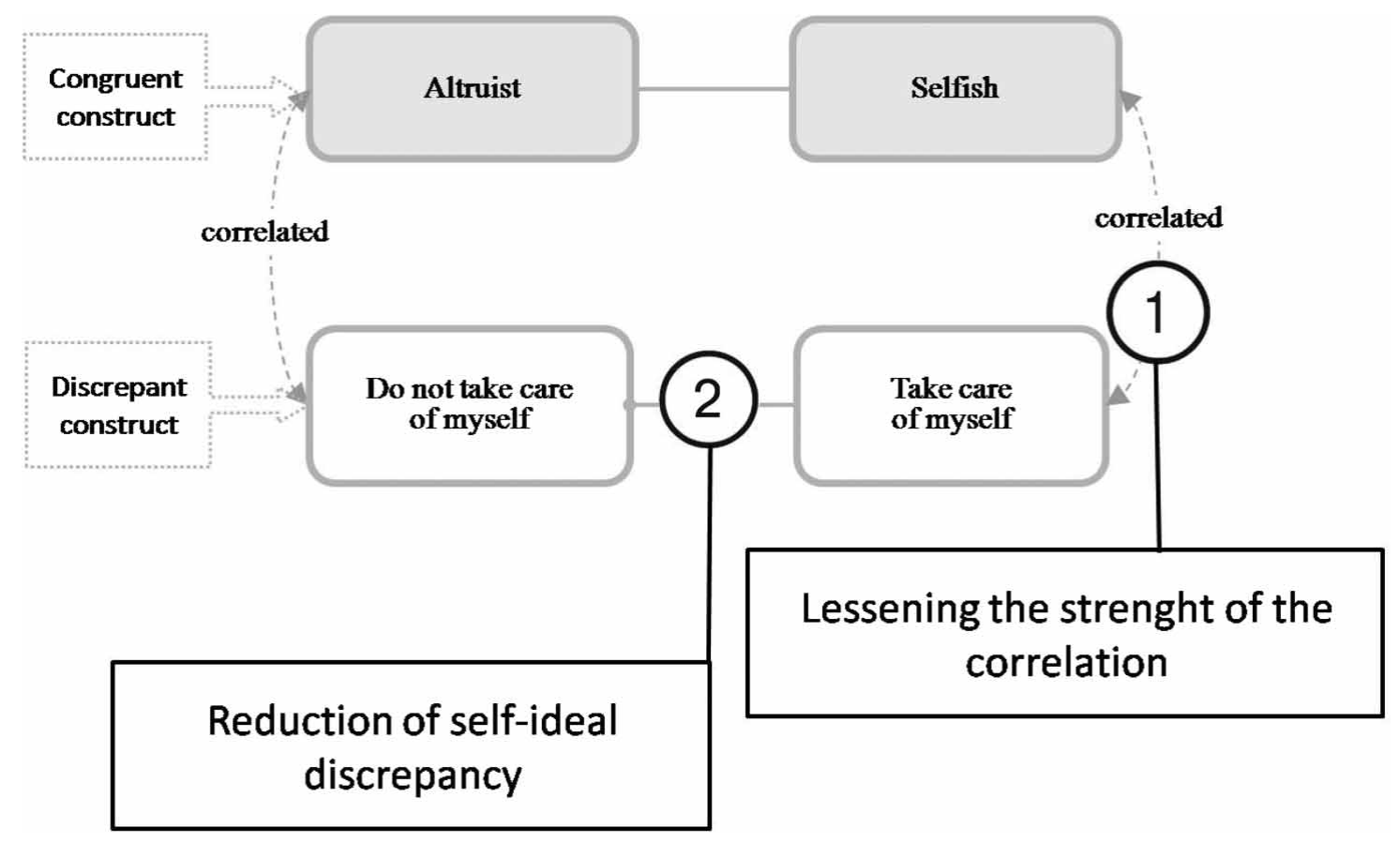

Figure 2. Hypothesized pathways for resolving IDs.

\section{Research Questions for the Current Study}

In this paper, we aim to shed light on change processes after a tailored dilemma-focused intervention for depression. We sought to investigate the way in which IDs are resolved and how such resolution is reflected in clients' accounts of change and their self-narratives at posttreatment, comparing two groups: Good and poor outcome participants. To that end, we used three instruments (see the Method section) to observe differences between groups: (i) the Repertory Grid Technique to track the resolution of dilemmas after therapy, (ii) the Change Interview to compile clients' account of changes at posttreatment and to compare betweengroup differences in the number and relevance of reported changes, and (iii) the Innovative Moments Coding System to examine the levels of self-narrative reconstruction (high-level vs. low-level IMs) at the Change Interview in each group.

It was expected that in the poor outcome group, IDs would not be resolved and that clients would present low-level IMs but not higher-level IMs. Consequently, clients will report a lower number of changes with less importance for their lives. By contrast, we expected that in the good outcome group, pretreatment IDs may be resolved after therapy, participants will present significantly more high-level IMs, and they will report a greater number of relevant changes at posttreatment. We also expected that a greater level of self-narrative reconstruction will be associated with greater magnitude of symptom improvement, as measured by the Beck Depression Inventory, second edition (Beck, Steer, \& Brown, 1996). In addition, we explored whether participants resolved their IDs according to the specific hypothesis noted above (Figure 2). Therefore, in the current study we examined the following research questions:

(1) Do the cases from the good outcome group present a greater level of conflict resolution than cases from the poor outcome group? If so, how do participants resolve their dilemmas?

(2) Do cases from the good outcome group report a greater number of relevant changes at posttreatment?

(3) Do the good outcome cases present a higher percentage of high-level IMs than the poor outcome cases?

(4) Is there a relationship between the magnitude of symptom improvement and the proportion of high-level IMs and IDs at posttreatment?

\section{Method}

\section{Participants}

Ten participants were selected from a pool of 22 clients seen as part of the dilemma-focused intervention arm of an ongoing study of Feixas et al. (2013, 2015). This is a randomized controlled clinical trial of psychotherapy for adults with depressive disorders that explore the relative efficacy of a dilemma-focused 
module intervention over standardized CBT. Inclusion criteria for the trial were as follows: (i) clients were over 18 years old, (ii) clients met diagnostic criteria for major depressive disorder or dysthymic disorder (APA; DSM-IV-TR, 2000), (iii) clients scored 19 or above on the Beck Depression Inventory (BDI-II; Beck et al., 1996), and (iv) clients presented at least one cognitive conflict, as assessed using the Repertory Grid Technique. Exclusion criteria were: (i) the presence of bipolar disorders, psychotic symptoms, substance abuse, organic brain dysfunction, or mental retardation; (ii) acute suicidal ideation; and (iii) current receipt of psychological treatment.

Participants who displayed the best five and the worst five outcomes were allocated into the good outcome and the poor outcome groups, respectively. Participants' type of outcome was established according to reliable change index criteria (Jacobson \& Truax, 1991) based on their BDI-II pretreatment and posttreatment scores (see Table I). We used Spanish normative data (Sanz \& Vázquez, 2011) for calculating the cut-off (16.92) and values for the reliable change index (9.17). Considering these two criteria, each case was classified as either good or poor outcome at the termination of therapy (see Table I).

Participants from the good outcome group showed a mean age of $50.00(S D=6.00)$; the group was formed by four women and one man. Four participants were medicated with psychotropic medications at intake (two took antidepressants; two took anxiolytics; and one took both medications). Four participants met the criteria for a co-morbid diagnosis, three for panic disorder without agoraphobia, and one for posttraumatic stress disorder. Three out of the five participants from this group had been in psychotherapy before. On the other hand, the poor outcome group yielded a mean age of $52.40(S D=10.45)$ and was constituted only of women. All of the participants were medicated with antidepressants; one was also medicated with an anxiolytic. Two of them had received psychotherapy previously. Three participants received a co-morbid diagnosis of panic disorder without agoraphobia, one posttraumatic stress disorder, and the other dysthymia. The participants from both groups displayed similar rates of treatment attendance: $89.6 \%$ for the good outcome group and $85 \%$ for the poor outcome group.

The therapists were all postgraduate students who were enrolled in the master's degree program in Cognitive Social Therapy at the University of Barcelona, Spain. The program is a three-year clinical training program that is grounded on a cognitive-constructivist framework. Therapists were also specifically trained in dilemma-focused intervention protocol and received supervision after each session to support their work in the trial and to ensure adherence to the manual.

\section{Instruments and Measures}

Structured Clinical Interview for DSM-IV Axis I Disorders (SCID-I; First, Spitzer, Gibbon, \& Williams, 1996). The SCID-I is a semi-structured interview for the diagnosis of categorically defined DSM-IVTR Axis I psychiatric disorders. SCID-I diagnoses display adequate test-retest reliability for depressive disorders in major depression $(K=.66$; Lobbestael, Leurgans, \& Arntz, 2011). Information regarding diagnosis and socio-demographic data were gathered for each participant.

Beck Depression Inventory-II (BDI-II; Beck et al., 1996). The BDI-II is a 21 -item self-report measure of depression that has demonstrated a strong internal consistency in psychiatric outpatients (.92). It was used as the main outcome measure for monitoring the severity of depressive symptoms. The Spanish version of the inventory was validated by Sanz and Vázquez (2011) and showed appropriate psychometric properties.

Repertory Grid Technique. This technique is a constructivist assessment procedure that was originally proposed by Kelly (Fransella, Bell, \& Bannister, 2004; Kelly, 1955/1991). It is a semi-structured interview that we used in our study to assess the presence and number of IDs. The interview is aimed to elicit personal constructs and their assessment as they are applied to a set of elements such as present self, ideal self, and significant others (see Feixas, Saúl, \& Ávila, 2009 for a detailed description of the format that was used in this study). The administration consists of three main stages. First, participants identified a set of significant others from their interpersonal world who, along with the present self and the ideal self, constituted the elements of the grid (columns). Second, the constructs (rows) were elicited by questions about similarities and differences between pairs of elements (e.g., how are your mother and father alike? How are they different?). For each response, an opposite construct pole was also elicited (e.g., what would be the opposite of this characteristic?). Finally, participants rated each element on the grid on each construct using a seven-point Likert scale ranging from very much like the right pole of the construct to very much like the left pole of the construct. Two measures were used for the assessment of conflicts using the software GRIDCORE v. 4.0 (Feixas \& Cornejo, 2002): (i) the presence or absence of IDs and (ii) the proportion of IDs. This proportion reveals the number of IDs in a 
Table I. Participants' outcome and the presence of IDs after therapy.

\begin{tabular}{|c|c|c|c|c|c|}
\hline Diagnosis & & BDI-II pre & BDI-II post & Status & $\begin{array}{c}\text { Dilemma } \\
\text { posttreatment }\end{array}$ \\
\hline \multirow[t]{5}{*}{ Poor outcome } & $\mathrm{r}$ & 40 & 50 & Deteriorated & No \\
\hline & $\mathrm{r}$ & 40 & 45 & Unchanged & Yes \\
\hline & $\mathrm{s}$ & 45 & 48 & Unchanged & Yes \\
\hline & $\mathrm{s}$ & 54 & 54 & Unchanged & No \\
\hline & $\mathrm{s}$ & 52 & 50 & Unchanged & Yes \\
\hline \multirow[t]{5}{*}{ Good outcome } & $\mathrm{s}$ & 41 & 25 & Improved & No \\
\hline & $\mathrm{s}$ & 43 & 14 & Recovered & No \\
\hline & $\mathrm{s}$ & 35 & 6 & Recovered & No \\
\hline & $\mathrm{r}$ & 27 & 6 & Recovered & Yes \\
\hline & $\mathrm{r}$ & 48 & 23 & Improved & No \\
\hline
\end{tabular}

Note. r, recurrent major depression; s, major depression single episode; BDI-II, Beck Depression Inventory, second edition.

participant's grid, taking into account its size, given that the number of constructs that are elicited during Repertory Grid administration varies across participants. This measure is calculated by dividing the number of dilemmas of a grid by the total number of possible combinations of constructs of such grid taking two at a time (and multiplied by 100 to calculate the percentage).

The Client Change Interview (Elliott et al., 2001). This is a semi-structured posttreatment qualitative interview that is designed to assess possible causal links between the work carried out in therapy and the changes, as perceived by the client. The interview attempts to work against the likelihood that psychotherapy is helpful and that client change is primarily due to the treatment (Elliott, 2002). In so doing, the interview deliberately seeks information about negative changes, hindering or missing factors as well as non-therapy factors that are related to change (Elliott, 2012). The interview is used to capture clients' subjective experience of change and gathers information about eight main topics: General experience of therapy, changes experienced, change ratings (expectedness, likelihood without therapy, and importance of each change within a five-point rating scale), attributions, personal resources, contextual limitations, helpful aspects of the therapy, and problematic aspects of the treatment. However, the interview uses open-ended explanatory questions, and clients are asked to provide as much detail as possible to provide an individually tailored assessment of their perception of change at the end of the therapy. In the present study, we used this interview in two ways: To compare between-group differences in reported changes (number of changes, expectedness, likelihood without therapy, and importance of each change) and to analyze the presence of IMs.

Innovative Moments Coding System (Gonçalves, Ribeiro, Mendes, et al., 2011). This is a qualitative method that was designed to track IMs in therapy sessions and interviews. The system is composed of five mutually exclusive categories of IMs (see Appendix 1): Action, reflection, protest, reconceptualization, and performing change. Gonçalves and colleagues (e.g., Gonçalves, Ribeiro, Mendes, et al., 2011) reported a high reliability index (kappa ranging from .80 to .97) on the overall IMs proportion. In our study, the system was used to code the type and proportion of IMs in the Clients' Change Interview. Proportion refers to the percentage of words of each IM's types in the transcript of the interview. For each group, we computed the mean proportion of low-level IMs (action, reflection, and protest) and high-level IMs (reconceptualization and performing change).

\section{Procedure}

Participants of Feixas et al.'s (2013) ongoing randomized clinical trial were recruited through medical referrals from several health care centers in the city of Barcelona, Spain. They were contacted to begin the assessment phase in their respective centers, where two independent and specifically trained master's-level students administered the SCID-I and the BDI-II in the first meeting to determine if clients met the inclusion criteria. A second appointment was then scheduled to complete the Repertory Grid Interview. All participants provided informed consent on forms that were approved by local research ethics committees. Once assessed, the clients started the 16-session treatment protocol. Individual sessions were audio recorded, as set forth in the informed consent agreement. A second assessment of all initial measures was carried out within one month after the termination of therapy by two independent evaluators who were unaware of the therapeutic condition of the participant. At that time, the 
Client Change Interview was administered and audio recorded for further analysis.

To acquire the sample of our study, we selected the best five and worse five completers out of 22 participants from the clinical trial database based on the reliable change index criteria, as stated in the participants section (pre-post changes on BDI-II scores). Using the software GRIDCORE v. 4.0, data from the Repertory Grid Technique were analyzed to obtain the two measures of conflict: Presence and the proportion of IDs (level of conflict).

Next, Client Change Interviews of the 10 participants were transcribed to track IMs. For the analysis of the IMs, two judges were assigned to rate the recordings of the Client Change Interview of each participant. The judges were two female master's-level therapists with one and seven years of clinical practice, respectively. They were trained for more than $20 \mathrm{hrs}$ using interviews from other cases until their ratings reached the expected reliability $(K>.80)$. The coders were aware of neither the outcome of each participant nor the research questions of the research. The analysis of IMs requires two different phases (see Gonçalves, Ribeiro, Mendes, et al., 2011 for a detailed description). First, evaluators must reach a consensual definition of the features of a client's problematic self-narrative. Thus, based on the sessions, the judges list a set of problems that are close to what emerged in the sessions (e.g., difficulties asserting own needs). Second, based on the previous definition, they proceed with the identification and categorization of each IM within the text in terms of type and proportion. For the first phase, in this study, the coders used three different sources of information to establish the features of problematic self-narrative of each client: (i) a brief report of the case made by the psychotherapist describing core complaints, (ii) the audio recordings of the two first sessions, and (iii) the ID on which the therapist focused the intervention. Once coders agreed on the features of the problematic self-narrative of each client, they independently coded the Client Change Interview of the participants using the recording and the transcripts. Finally, their coding was supervised by the research manager to ensure adherence to the coding system. Reliability indexes were calculated for both the overall proportion of IMs (percentage of agreement $=88.3 \%$ ) and IM type $(K=.92)$, which indicated a high degree of interjudge reliability.

In sum, the Repertory Grid Technique and the BDI-II were completed at baseline and again at posttreatment, whereas the Client Change Interview was completed only at posttreatment. IDs were derived from the Repertory Grid analysis (pre- and posttreatment), and the Clients Change Interview was coded for IMs (posttreatment).

\section{Treatment}

The treatment of the dilemma-focused arm of the trial consisted in the application of an eight-session tailored individual dilemma-focused intervention based on the manual by Feixas and Compañ (2015). This individual protocol uses IDs as a central issue for case formulation. Therefore, in the first session, therapists and clients discussed the identified dilemma, conjointly evaluating the pertinence of addressing such conflict to achieve therapeutic goals. The focus of the second session was on the elaboration of the dilemma, inspecting the personal meanings and implications of the congruent and discrepant constructs using specific techniques (e.g., laddering, Tschudi's ABC model). In the third session, the therapist is intended to address the influence of the ID in the client's everyday life using the cycle of experience as a guiding concept. The fourth and fifth sessions focused on the historic reconstruction of the dilemma, with special attention to the relational implications of the dilemma. A tailored version of the two-chair technique is used in session six to facilitate the resolution of the dilemma. The final session aimed at developing and validating a non-dilemmatic self-narrative working, among other things, on the expansion of unique outcomes. One extra session could be used to supplement any of the above-mentioned issues as needed.

\section{Results}

The number of participants in each group of the present study $(n=5)$ precluded using parametric tests. Therefore, we mainly used a nonparametric approach to test our research questions.

Do the cases from the good outcome group present a greater level of conflict resolution than cases from the poor outcome group? If so, how do participants resolve their dilemmas?

There were no significant between-group differences in the level of conflict at pretreatment $(U=$ $12 ; p=.92$ ), and only the good outcome group significantly reduced $(z=-2.03, p=.04)$ the proportion of IDs after the therapy, with a medium effect size $(r=.45)$. Indeed, only one participant from the good outcome group presented with IDs at posttreatment, whereas three of the five in the poor outcome did so (see Table I) and in greater proportion (see Table II).

We inspected the posttreatment repertory grid data to explore whether participants resolved IDs according to the specified hypothesis (Figure 2). From the initial 18 IDs presented, 15 were resolved. In 9 of the 15 resolved dilemmas, the self-ideal discrepancy 
Table II. Between-group differences in symptoms, proportion of dilemmas, number and characteristics of changes, and proportion of low and high IMs.

\begin{tabular}{lcc}
\hline & $\begin{array}{c}\text { Good outcome } \\
\text { group mean }(S D)\end{array}$ & $\begin{array}{c}\text { Poor outcome } \\
\text { group mean }(S D)\end{array}$ \\
\hline BDI-II scores & & $46.20(6.57)$ \\
Pretreatment & $38.8(8.07)$ & $49.4(3.28)$ \\
Posttreatment & $14.80(9.04)$ & $2.67(2.96)$ \\
Proportion of IDs & & $3.40(4.41)$ \\
Pretreatment & $1.73(1.19)$ & 20 \\
Posttreatment & $0.26(0.59)$ & $2-6$ \\
Client Change Interview & & 22 \\
Total number of changes & $2-7$ & $4.0(2.0)$ \\
Minimum-maximum per person & $4.4(1.8)$ & $4.2(0.8)$ \\
Number of changes & $3.2(1.3)$ & $1.3(0.5)$ \\
Expectedness of changes & $1.5(0.8)$ & $4.4(0.5)$ \\
Likelihood without therapy & $4.5(0.5)$ & $17.83(15.41)$ \\
Importance of changes & & $3.02(6.74)$ \\
Type of IMs & $19.03(15.09)$ & $21.34(13.19)$ \\
Low-level IMs (ARP) & & \\
High-level IMs (RCPC) & & \\
\hline
\end{tabular}

Note. BDI-II, Beck Depression Inventory, second edition; SD, standard deviation; IMs, innovative moments; ARP, action, reflection, and protest IMs; RCPC, reconceptualization and performing change IMs.

on the discrepant construct at intake was lessened to the extent of no longer being discrepant at posttreatment, whereas it continued to be discrepant but the association with the congruent construct was diminished in six instances. There was no case in which the resolution of ID was based on the rupture of the self-ideal congruency.

Do cases from the good outcome group report a greater number of relevant changes at posttreatment?

As explained before, information regarding the number and relevance of changes after treatment was obtained by means of the Client Change Interview. During administration, the interviewer compiled the expressed changes in a list, and clients rated each change on a five-point Likert scale with regard to their expectedness, likelihood of occurrence without therapy, and the relevance for their lives. A series of Mann-Whitney $U$ tests revealed no significant between-group differences in the clients' ratings (expectedness: $U=7.5 ; p=.29$; likelihood without therapy: $U=11 ; p=.74$; importance: $U=$ $12 ; p=.91)$ or in the number of changes $(U=11 ; p$ $=.75$ ) of each group. As shown in Table II, participants from both groups displayed a mean of approximately four changes after therapy and, although they were not extremely surprised, participants experienced changes as being unlikely to occur without therapy and very important for their lives.

Do the good outcome cases present a higher percentage of high-level IMs than the poor outcome cases?

To check between-group differences in the proportion of low- and high-level IMs, two MannWhitney U tests were conducted using a Bonferroni adjusted alpha level of $.025(.05 / 2)$. Whereas there were no significant differences in the proportions of low-level IMs $(U=10, z=-0.52, p=.60)$, the good outcome group showed a significantly greater proportion of high-level IMs than the poor outcome group $(U=2, z=-2.263, p=.024$; see Table II), with a large effect size $(r=.71)$.

Is there a relationship between the magnitude of symptom improvement and the proportion of high-level IMs and IDs at posttreatment?

Given the previous results, we considered inspecting the association of the magnitude of symptom improvement with the proportion of high-level IMs and the proportion of IDs after therapy. To that end, we used the difference between the pre- and posttreatment BDI-II scores of each participant (Jacobson \& Truax, 1991) as a measure of the magnitude of symptom improvement. Spearman's correlations coefficients revealed a significant strong correlation between the magnitude of symptom improvement and the proportion of high-level IMs (rho $=.73, n=$ $10, p=.02)$ and a medium negative correlation with the proportion of IDs $(r=-.40, n=10, p=.24)$.

\section{Discussion}

The present study examined change in the self-narrative of depressive clients after dilemma-focused therapy. We used three instruments, the Repertory Grid Technique, the Client Change Interview, and the Innovative Moments Coding System, to observe differences in self-construing and dilemmas 
between good and poor outcome cases of an ongoing trial (Feixas et al., 2013). In accordance with previous research (Feixas, Saúl, Winter, \& Watson, 2008), participants from the good outcome group showed a significant reduction in the presence and proportion of conflicts. These findings are only preliminary and should be treated as such but nevertheless suggested that a clinically significant change is associated with the resolution of IDs, given that the groups did not differ on baseline measures at intake. Moreover, the magnitude of symptom improvement was moderately associated with the resolution of conflicts. Indeed, whereas in the good outcome group, only one participant presented with dilemmas after therapy and in a smaller proportion, the poor outcome group increased their rate of IDs. These results suggested that, at least for those clients who presented with IDs, recovery is associated with the resolution of conflicting configurations of the self.

To the best of our knowledge, this is the first empirical study that examined the way in which clients resolve IDs after psychotherapy. Results from this study supported our hypothesized pathway for conflict resolution (Figure 2). In particular, we found that the participants resolved their dilemmas by loosening the association between congruent and discrepant constructs and by reducing self-ideal discrepancy. In no case did self-ideal congruency change. From a clinical viewpoint, these results suggest that symptom improvement is associated not only with the enhancement of self-esteem (understood as a reduction of self-ideal discrepancy) but also with the resolution of inner conflicts, even when ideal-self discrepancy reduction is not achieved. Therefore, clinicians should not only consider helping clients to achieve change but also to reconcile the need for change with the need for coherence in their sense of identity. In narrative therapy terms (White, 2007; White \& Epston, 1990), the alternative self-narrative should be anchored to core aspects of a client's sense of identity to assure its continuity and the client's sense of agency and authorship. This approach could constitute a fruitful therapeutic strategy to create meaning bridges (Stiles, 2011) in the face of competing plotlines (Boritz, Bryntwick, Angus, Greenberg, \& Constantino, 2014).

A surprising finding was that participants from both groups did not differ in the number, perceived relevance, and expectancy of reported changes at post-therapy, as evaluated through the Client Change Interview. Rather, our results suggested that therapy prompted relevant changes, as perceived by the clients, in both groups. Interestingly, these changes were underestimated by standard outcome measures. This finding is also consistent with the occurrence of IMs in both groups, despite the difference between low- and high-level types of IMs. However, the groups differed in the degree to which such changes were integrated into a new self-view and a reconstructed self-narrative, as revealed by the analysis of IMs. In accordance with the existing research (e.g., Gonçalves, Ribeiro, Mendes, et al., 2011), the groups displayed specificity in their IMs profiles. Whereas both groups showed equally lowlevel IMs (action, reflection, and protest IMs), the good outcome group reported a significantly higher proportion of high-level IMs (reconceptualization of the self and performing change IMs). Moreover, there was a strong correlation between the magnitude of symptom improvement and the proportion of highlevel IMs at posttreatment.

Taken together, our results showed that clinically significant change and symptom improvement were associated with the resolution of dilemmas and a higher rate of reconceptualization and performing change IMs in the clients' self-narratives. An inspection of the transcripts from the Client Change Interviews provided some illustrative excerpts of the association between high-level IMs and the resolution of dilemmas. The following fragment is taken from the interview of the client whose ID is represented in Figure 1 in the introduction section:

This therapy has had a good impact on me because I begin again to ... to realize that I have to think more about myself [a move on the discrepant construct fostering an alternative self-narrative] because I was always thinking about the needs of others and pushing my things into the background, virtually consigned into oblivion. You know I used to always place others before myself. For instance, if someone came to me and said, "Do you mind coming with me to ..." or "could you do that or whatever...", I always said "yes" no matter the price [description of past problematic self-narrative]. I realized that this was a way - not the best way after all - to try to be a good person [description of the conflictual association]. However, that "me" is finished. No more servitude as a virtue. I do know now where the limits are [first component of the reconceptualization IM: description of the contrast between problematic and alternative self narrative]. Now, I take care of myself first and look after my things, my work, my paintings and my feelings... without feeling guilty but proud of it [second component of the reconceptualization IM: description of the process which allowed for change].

As highlighted in brackets in the above text, we found all of the elements of reconceptualization IM in relation to the targeted ID. The client moved toward the desired pole of her discrepant construct, "taking care of myself." Such movement represented the emergence of a new self-narrative in contrast with the problematic self-view of "not taking care of 
herself." The client demonstrated being aware that such a position was, in a certain manner, a manifestation of one of her core values: Being a good person. Eventually, she developed a new position from which taking care of herself does not imply any threat to her identity and thus facilitates the crystallization of a renovated self-narrative. This is further developed in the interview, for instance, when the participant was asked about her self-description:

Now I am the kind of person who knows where the limits are. I think more about what I have to do, placing my needs and the things I have to do before others' needs or demands. It is true that sometimes I still go out of my way, but I realize that I do it less than before, you know? Now I am more in the line, I think, I have to be, I can be sensitive with others without losing myself; otherwise where is the limit ... suicide?

In this excerpt, it can be observed how the resolution of the dilemma is reflected in the client's selfnarrative. Here, "sensitive with others" reflects her position in the pole of the congruent construct "altruistic" (vs. selfish), whereas "losing myself" can be associated with the pole "not taking care of herself" (vs. taking care of herself) of the discrepant construct. It can be clearly seen how she was able to reconceptualize her self, breaking her previous dilemmatic association between "taking care of herself" and "becoming selfish" and, therefore, simultaneously becoming able to "taking care of herself" and preserving her core identity construction of being "altruistic" (vs. selfish).

It is important to note, however, that IDs are not easy to track in a client's self-narrative, given IDs' implicit nature as deep self-schemas. Indeed, detecting such tacit schemas requires the administration of specific instruments such as the Repertory Grid. During psychotherapy, IDs can be accessed by identifying problematic reaction points (Greenberg, Rice, \& Elliot, 1993) that potentially involve the constructs that form the dilemma and interfere with a more adaptive and satisfying functioning.

In sum, the results from our study suggested that recovery in dilemma-focused therapy for depression is probably associated with the resolution of IDs (at least in clients with ID at the beginning of therapy) and that the resolution of these identity-related conflicts is, to a great extent, associated with the emergence of a non-dilemmatic self-narrative. However, it is also worth noting that for some individuals in our sample, a significant change in symptoms was not associated with the resolution of IDs, which lessens the evidence for the mediating role of this change mechanism. For instance, the only participant who deteriorated showed no ID at posttreatment, and a client from the good outcome group did not resolve her ID during the therapy. Undoubtedly, there are other influential variables (e.g., alliance, emotional processing, life events, medication) that affect outcome effects that we did not control in our study. Future research is needed to explore the relative influence of conflict resolution for the process of change, taking into account other key factors.

Our results are in line with the body of research that supports the mediating role of change in "deep" cognitive schemas for therapeutic improvement in depression (Dozois \& Dobson, 2001; Garratt, Ingram, Rand, \& Sawalani, 2007). Furthermore, the results of this study are also consistent with other therapeutic models that highlight internal conflicts and/or self-narrative reconstruction in relation to the change processes of depressive clients. For example, the motivational interviewing model upholds that depressed clients tend to be ambivalent toward change. Decisional balance then becomes a preferred intervention (Arkowitz \& Miller, 2008). Emotion-focused therapy has developed sophisticated techniques for helping clients to dialog between two conflicting aspects of the self (e.g., the two-chair technique), to achieve deeper emotional processing, which has been shown to predict good treatment outcomes (Greenberg \& Pascual-Leone, 2006). In this vein of research, the assimilation model (Stiles, 2001) has demonstrated that, in depression, a significant change is associated with the integration of conflicting voices (Osatuke, Stiles, Barkhem, Hardy, \& Shapiro, 2011). Moreover, recent research about IMs in emotion-focused therapy for depression has identified specific narrative markers that are associated with difficulties in consolidating a reconstructed self-narrative (Ribeiro et al., 2014). Additionally, coherence therapy (e.g., Ecker \& Hulley, 2008) has indicated that symptom maintenance is linked to the need for preserving the coherence of self-identity.

Regardless of the actual model that is adopted, our findings could also be seen as partial empirical support for these types of models, which address ambivalence and conflict in the psychotherapeutic process. Given the limited design of this study, we failed to assess treatment specificity. Future research might explore whether conflict resolution and selfnarrative transformation are common mechanisms in several conditions and psychotherapy approaches or specific to depression and certain therapeutic techniques. This would help us to advance our knowledge on the controversial cognitive specificity hypothesis for depression (Garratt et al., 2007).

An innovative feature of our study was to analyze a medium-sized set of data from the Client Change 
Interview of multiple cases using the IMs coding system. This method has frequently been used to track change throughout the therapy process but not at posttreatment interviews. In our opinion, the Innovative Moments Coding System was sensitive enough to capture change from the Client Change Interview and was more accurate in detecting group specificity than the Client Change Interview rating scales per se. Therefore, the Innovative Moments Coding System demonstrated its usefulness in analyzing cross-sectional psychologically relevant texts, and, in combination with the Client Change Interview, it provides an invaluable opportunity to understand the process of change. Interestingly, the findings that we obtained in the interviews were consistent with the results found in tracking IMs along treatment, as high-level IMs seem to characterize good outcome cases.

\section{Limitations}

Apart from the shortcomings noted above, there are other relevant limitations that need to be recognized when interpreting these results and considering their clinical implications. First and foremost, the small sample size precluded parametric tests and limited further analysis of associations between variables. Second, the study is correlational in nature, and therefore no causal relationships can be established between symptom improvement and self-narrative changes. Third, groups were formed according to their outcome at post-therapy, but future research should replicate the findings while also taking into account follow-up data. Finally, we used a posttreatment interview to examine clients' accounts of change and the analysis of IMs, but we were unable to track when and exactly how these changes occurred. However, as stated above, the way in which IMs are coded ensures that they are truly novelties because they are identified in contrast to a consensual definition of the features of the client's problematic selfnarrative at intake. Future research should focus on important episodes of therapeutic change in dilemma-focused therapy to understand how clients' processes are facilitated, by which therapist interventions, and under what particular contexts.

\section{Clinical Implications}

Despite the methodological limitations, our findings lead us to some important clinical implications. In light of our results, it seems reasonable to assume that targeting IDs in the therapy context may help clients to achieve a greater level of reconceptualization and change inasmuch as the dilemma-focused intervention is specifically designed to reorganize self-representations in conflict. In doing so, not only is the negative self-schema (discrepant constructs) reconceptualized, but positive attributes (congruent constructs) are also reinforced and elaborated, facilitating wholeness and integration of the self, which may eventually contribute to diminishing the likelihood of relapse (Dozois \& Dobson, 2001). Further, focusing on IDs might help in personalizing the design of a treatment to fit a client's subjective experiences and needs and, in turn, resolving ambivalence toward change. Certainly, in clients who experience difficulties overcoming their symptoms, psychotherapists could benefit considerably from screening for the presence of IDs, as it could enhance case formulation and shed light on the personal factors that maintain or worsen the disorder.

\section{Funding}

This study has been partially supported by the Portuguese Foundation for Science and Technology (PTDC/ PSI-PCL/121525/2010) and the Spanish Ministry of Economy and Competitiveness (PSI2011-23246).

\section{References}

American Psychiatric Association. (2000). Diagnostic and statistical manual of mental disorders (4th ed., rev.). Washington: Author.

Arkowitz, H., \& Miller, W. R. (2008). Learning, applying and extending motivational interviewing. In H. Arkowitz, H. A. Westra, W. R. Miller, \& S. Rollnick (Eds.), Motivational interviewing in the treatment of psychological problems (pp. 1-25). New York: Guilford.

Barth, J., Munder, T., Gerger, H., Nüesch, E., Trelle, S., Znoj, H., ... Cuijpers, P. (2013). Comparative efficacy of seven psychotherapeutic interventions for patients with depression: A network meta-analysis. PLoS Medicine, 10, e1001454. Retrieved from http://www.plosmedicine.org/article/Authors/ info:doi/10.1371/journal.pmed.1001454

Basto, I., \& Salgado, J. (2014). La investigación sobre el proceso de cambio en psicoterapia y los diferentes enfoques terapéuticos: un análisis de los mecanismos cognitivos y emocionales [Research on the process of change in psychotherapy and the different therapeutic approaches: An analysis of cognitive and emotional mechanism]. Revista de Psicoterapia, 25(99), 31-47.

Beck, A. T., Steer, R. A., \& Brown, G. (1996). Beck Depression Inventory II Manual. San Antonio, TX: The Psychological Corporation.

Boritz, T. Z., Bryntwick, E., Angus, L., Greenberg, L. S., \& Constantino, M. J. (2014). Narrative and emotion process in psychotherapy: An empirical test of the Narrative-Emotion Process Coding System (NEPCS). Psychotherapy Research, 24, 594-607.

Cuijpers, P., Andersson, G., Donker, T., \& Van Straten, A. (2011). Psychological treatment of depression: Results of a series of meta-analyses. Nordic Fournal of Psychiatry, 65, 354-364. 
Cunha, C., Gonçalves, M. M., Hill, C. E., Mendes, I., Sousa, I., Ribeiro, A. P., ... Greenberg, L. (2012). Therapist interventions and client innovative moments in emotion-focused therapy for depression. Psychotherapy, 4, 536-548.

Dozois, D. J. A., \& Dobson, K. S. (2001). A longitudinal investigation of information processing and cognitive organization in clinical depression: Stability of schematic interconnectedness. Fournal of Consulting and Clinical Psychology, 69, 914-925.

Ecker, B., \& Hulley, L. (2008). Coherence therapy. In J. D. Raskin \& S. K. Bridges (Eds.), Studies in meaning (Vol. 3, pp. 57-84). New York: Pace University Press.

Elliott, R. (2002). Hermeneutic single case efficacy design. Psychotherapy Research, 12, 1-20.

Elliott, R. (2012). Qualitative methods for studying psychotherapy change process. In A. Thomson \& D. Harper (Eds.), Qualitative research methods in mental health and psychotherapy: An introduction for students $\mathcal{G}$ practitioners (pp. 69-81). Chichester: WileyBlackwells.

Elliott, R., Slatick, E., \& Urman, M. (2001). Qualitative change process research on psychotherapy: Alternative strategies. In J. Frommer \& D. L. Rennie (Eds.), Qualitative psychotherapy research: Methods and methodology (pp. 69-111). Lengerich: Pabst Science Publishers.

Engle, D., \& Arkowitz, H. (2008). Viewing resistance as ambivalence: Integrative strategies for working with resistant ambivalence. Fournal of Humanistic Psychology, 48, 389-412.

Feixas, G., Bados, A., García-Grau, E., Montesano, A., Dada, G., Compañ, V.,... Winter, D. (2013). Efficacy of a dilemmafocused intervention for unipolar depression: Study protocol for a multicenter randomized controlled trial. Trials, 14 (144). Retrieved from http://www.trialsjournal.com/content/14/1/144

Feixas, G., Bados, A., Garcia-Grau, E., Paz, C., Montesano, A., Compañ, V., ... Medeiros, L. y otros. (2015, June). Eficàcia d'una intervenció cognitiva centrada en dilemes per a la depressió. [Efficacy of a cognitive dilemma-focused intervention for depression]. Paper presented at Societat Catalana de Psiquiatria i Salut Mental, Barcelona.

Feixas, G., \& Compañ, V. (2015). Manual de intervención centrada en dilemas para la depresión [Manual of a dilemma-focused intervention for depression]. Bilbao: Desclée de Brouwer.

Feixas, G., \& Cornejo, J. M. (2002). Correspondence analysis for grid data [Computer Software] and Reperory Grid Manual. Barcelona: Psimedia.

Feixas, G., Montesano, A., Compañ, V., Salla, M., Dada, G., Pucurull, O., ... Guardia, J. (2014). Cognitive conflicts in major depression: Between desired change and personal coherence. British Fournal of Clinical Psychology. doi:10.1111/bjc. 12050

Feixas, G., Montesano, A., Erazo-Caicedo, M. I., Compañ, V., \& Pucurull, O. (2014). Implicative dilemmas and symptom severity in depression: A preliminary and content analysis study. Fournal of Constructivist Psychology, 27(1), 31-40.

Feixas, G., Saúl, L., Winter, D., \& Watson, S. (2008). Un estudio naturalista sobre el cambio de los conflictos cognitivos durante la psicoterapia. Apuntes de Psicología, 26, 243-255.

Feixas, G., Saúl, L. A., \& Ávila, A. (2009). Viewing cognitive conflicts as dilemmas: Implications for mental health. Fournal of Constructivist Psychology, 22, 141-169.

First, M. B., Spitzer, R. L., Gibbon, M., \& Williams, J. B. W. (1996). Structured clinical interview for DSM-IV Axis I disorders, clinician version (SCID-CV). Washington: American Psychiatric Press.

Fransella, F., Bell, R., \& Bannister, D. (2004). A manual for repertory grid technique. London: John Wiley \& Sons.

Garratt, G., Ingram, R. E., Rand, K. L., \& Sawalani, G. (2007). Cognitive processes in cognitive therapy: Evaluation of the mechanisms of change in the treatment of depression. Clinical Psychology: Science and Practice, 14, 224-239.

Gonçalves, M. M., Matos, M., \& Santos, A. (2009). Narrative therapy and the nature of "innovative moments" in the construction of change. Fournal of Constructivist Psychology, 22, 123.

Gonçalves, M. M., \& Ribeiro, A. P. (2012). Therapeutic change, innovative moments, and the reconceptualization of the self: A dialogical account. International fournal for Dialogical Science, 6, 81-98.

Gonçalves, M. M., Ribeiro, A. P., Conde, T., Matos, M., Santos, A., Martins, C., \& Stiles, W. B. (2011). The role of mutual infeeding in maintaining problematic self-narratives: Exploring one path to therapeutic failure. Psychotherapy Research, 21, 2740.

Gonçalves, M. M., Ribeiro, A. P., Mendes, I., Matos, M., \& Santos, A. (2011). Tracking novelties in psychotherapy process research: The innovative moments coding system. Psychotherapy Research, 21, 497-509.

Greenberg, L. S., \& Pascual-Leone, A. (2006). Emotion in psychotherapy: A practice-friendly research review. Fournal of Clinical Psychology, 62(5), 611-630.

Greenberg, L. S., Rice, L. N., \& Elliott, R. (1993). Facilitating emotional change: The moment-by-moment process. New York: Guilford Press.

Hollon, S. D. (2006). Cognitive therapy in the treatment and prevention of depression. In T. Joiner, J. S. Brown, \& J. Kistner (Eds.), The interpersonal, cognitive, and social nature of depression (pp. 133-151). Mahwah, NJ: Erlbaum.

Jacobson, N. S., \& Truax, P. (1991). Clinical significance: A statistical approach to defining meaningful change in psychotherapy research. Fournal of Consulting and Clinical Psychology, 59, 12-19.

Kelly, G. A. (1955/1991). The psychology of personal constructs. New York: Routledge.

Kelly, G. A. (1969). The autobiography of a theory. In B. Maher (Ed.), Clinical psychology and personality: The selected papers of George Kelly (pp. 46-65). New York: Wiley.

Lobbestael, J., Leurgans, M., \& Arntz, A. (2011). Inter-rater reliability of the structured clinical interview for DSM-IV Axis I disorders (SCID I) and Axis II disorders (SCID II). Clinical Psychology \& Psychotherapy, 18(1), 75-79.

Montesano, A., Feixas, G., Erazo-Caicedo, M. I., Saúl, L. A., Dada, G., \& Winter, D. (2014). Cognitive conflicts and symptom severity in dysthymia: "I'd rather be good than happy”. Salud Mental, 37, 41-48.

Montesano, A., Feixas, G., Muñoz, D., \& Compañ, V. (2014). Systemic couple therapy for dysthymia. Psychotherapy, 51, 30-40.

Montesano, A., Feixas, G., \& Varlotta, N. (2009). Análisis de contenido de constructos personales en la depresión [Content analysis of personal constructs in depression]. Salud Mental, 32, 371-379.

Montesano, A., Feixas, G., \& Winter, D. (2014, submitted for publication). Depression and identity: Are self-constructions negative or conflictual?.

Osatuke, K., Stiles, W. B., Barkham, M., Hardy, G. E., \& Shapiro, D. A. (2011). Relationship between mental states in depression: The assimilation model perspective. Psychiatry Research, 190(1), 52-59.

Ribeiro, A., Bento, T., Gonçalves, M. M., \& Salgado, J. (2010). Self-narrative reconstruction in psychotherapy: Looking at different levels of narrative development. Culture \& Psychology, 16, 195-212.

Ribeiro, A. P., Mendes, I., Stiles, W. B., Angus, L., Greenberg, L., Sousa, I., \& Gonçalves, M. M. (2014). Ambivalence in emotion focused therapy for depression: The maintenance of problematically dominant self-narratives. Psychotherapy Research, 24(6), $702-710$. 
Rowe, D. (1983). Depression: The way out of your prison. London: Routledge and Kegan Paul.

Sanz, J., \& Vazquez, C. (2011). Adaptacion española del Inventario para de Depresion de Beck-II (BDI-II) [Spanish adaptation of Beck's Depression Inventory - II]. Madrid: Pearson Education. de Shazer, S. (1985). Keys to solution in brief therapy. New York: W. W. Norton \& Company.
Stiles, W. B. (2001). Assimilation of problematic experiences. Psychotherapy, 38, 462-465.

Stiles, W. B. (2011). Coming to terms. Psychotherapy Research, 21, 367-384.

White, M. (2007). Maps of narrative practice. New York: Norton.

White, M., \& Epston, D. (1990). Narrative means to therapeutic ends. New York: Norton. 
Appendix 1.

Categories of IMs with examples

\begin{tabular}{ll}
\hline & \multicolumn{1}{c}{ Contents } \\
\hline Action & New coping behaviors facing anticipated or existent \\
& obstacles; \\
- Effective resolution of unsolved problem(s); & - Active exploration of solutions; \\
- Restoring autonomy and self-control; \\
- Searching for information about the problem(s).
\end{tabular}

Reflection

Subtype I. Creating distance from the problem(s)

- Comprehension-Reconsidering problem(s)' causes and/or awareness of its effects;

- New problem(s) formulations;

- Adaptive self-instructions and thoughts;

- Intention to fight problem(s)' demands, references of self-worth, and/or feelings of well-being.

Subtype II. Centered on the change

- Therapeutic process-Reflecting about the therapeutic process;

- Change process-Considering the process and strategies; implemented to overcome the problem(s); references of self-worth and/or feelings of well-being (as consequences of change);

- New positions - references to new/emergent identity versions in face of the problem(s).

Protest Subtype I. Criticizing the problem(s)

- Repositioning oneself toward the problem(s).

Subtype II. Emergence of new positions

- Positions of assertiveness and empowerment.

Reconceptualization RC always involves two dimensions:

- Description of the shift between two positions (past and present);

- The process underlying this transformation.

Performing change

- Generalization into the future and other life dimensions of good outcomes;

- Problematic experience as a resource to new situations;

- Investment in new projects as a result of the process of change;

- Investment in new relationships as a result of the process of change;

C: I realize that what I was doing was just, not humanly possible because I was pushing myself and I never allowed myself any free time, uh, to myself ... and it's more natural and more healthy to let some of these extra activities go ...

C: Yesterday, I went to the cinema for the first time in months!

Examples
C: Yesterday, I went to the cinema for the first time in
months!

C: I believe that our talks, our sessions, have proven fruitful, I felt like going back a bit to old times, it was good, I felt good, I felt it was worth it.

C: What am I becoming after all? Is this where I'll be getting to? Am I going to stagnate here!?

C: I am an adult and I am responsible for my life, and I want to acknowledge these feelings and I'm going to let them out! I want to experience life, I want to grow and it feels good to be in charge of my own life

C: You know ... when I was there at the museum, I thought to myself: you really are different ... A year ago you wouldn't be able to go to the supermarket! Ever since I started going out, I started feeling less depressed ... it is also related to our conversations and changing jobs ...

$\mathrm{T}$ : How did you have this idea of going to the museum?

C: I called my dad and told him: we're going out today!

$\mathrm{T}$ : This is new, isn't it?

C: Yes, it's like I tell you ... I sense that I'm different ...

T: You seem to have so many projects for the future now! C: Yes, you're right. I want to do all the things that were impossible for me to do while I was dominated by depression. I want to work again and to have the time to enjoy my life with my children. I want to have friends again. The loss of all the friendships of the past is something that still hurts me really deeply. I want to have friends again, to have people to talk to, to share experiences and to feel the complicity in my life again

- Performance of change: New skills;

- Re-emergence of neglected or forgotten self-versions. 